\title{
The Role of Radiotherapy in the Treatment of Retroperitoneal Lymph Node Metastases from Colorectal Cancer
}

This article was published in the following Dove Press journal: Cancer Management and Research

\section{Pei Shu \\ Ganlu Ouyang \\ Fang Wang \\ Jitao Zhou \\ Yali Shen \\ Zhiping Li \\ Xin Wang}

Department of Abdominal Oncology, Cancer Center, West China Hospital, Sichuan University, Chengdu, Sichuan Province, People's Republic of China
Correspondence: Xin Wang Email wangxin213@sina.com
Purpose: Retroperitoneal lymph node metastases are rare in colorectal cancer. Optimal treatment strategies are still unknown.

Patients and Methods: We retrospectively enrolled colorectal cancer patients who had received radiotherapy for retroperitoneal lymph node metastases from 2009 to 2018. Patients with isolated retroperitoneal lymph node metastases or retroperitoneal lymph nodes with extra-retroperitoneal metastases were all included. A median dose of 60 Gy was delivered.

Results: A total of 68 patients were enrolled in this study; 28 (41\%) of them had extraretroperitoneal metastases. In the isolated retroperitoneal lymph node metastases group, complete response was found in 5 patients (12.5\%), partial response was achieved in 20 patients $(50 \%), 9$ patients $(22.5 \%)$ had stable disease. The 1-, 2- and 3-year local control rates were $87.5 \%, 77.5 \%$, and $70 \%$. In the extra-retroperitoneal metastases group, the disease control rate was $75 \%$, including complete response in 1 patient (3.6\%), partial response in 4 patients $(14.3 \%)$ and stable disease in 16 patients (57.1\%). The 1-, 2- and 3-year local control rates were $57.1 \%, 42.8 \%$, and $0 \%$. The median overall survival was 59.4 months and 19 months in the isolated retroperitoneal lymph node metastases group and extra-retroperitoneal metastases group, respectively. In the isolated retroperitoneal lymph node metastases group, the 1-year and 3-year overall survival values were $90.2 \%$ and $75.8 \%$, respectively. The 1 -year and 3-year progression-free survival values were $57.9 \%$ and $0 \%$, respectively. The extra-retroperitoneal metastases group experienced worse survival outcome (1-year overall survival: $57.9 \%, P<0.05$; and 1-year progression-free survival: $22.5 \%, P<0.05$ ).

Conclusion: For patients with isolated retroperitoneal lymph node metastases, radiotherapy combined with systemic treatment can be used as a method to achieve no evidence of disease and can result in good local control and survival. For patients with extra-retroperitoneal metastases, although the survival is much worse than that of isolated retroperitoneal lymph node metastases, radiotherapy is an effective palliative treatment to relieve pain and obstruction based on systemic treatment.

Keywords: radiotherapy, retroperitoneal lymph node, colorectal cancer

\section{Introduction}

Colorectal cancer is the third most commonly diagnosed cancer and the fourth cause of cancer death, with an estimated 693,900 deaths per year. ${ }^{1}$ Fifty to sixty percent of those

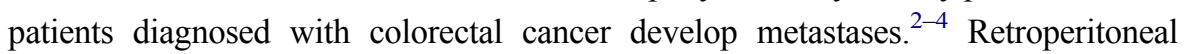
lymph node (RPLN) metastases are rare with a reported incidence of $1-2 \%$ and are often accompanied by metastases in other sites. ${ }^{5,6}$ The treatment of RPLN metastases remains controversial. 
Patients with colorectal cancer most commonly develop liver metastasis or lung metastasis, which is often presented as an oligometastatic disease. It is suggested by the European Society for Medical Oncology (ESMO) that for patients with oligometastatic disease, suitable localized intervention including surgery or radiotherapy should be recommended to achieve no evidence of disease (NED). ${ }^{7}$

If no treatment was delivered to those patients who had RPLN metastases, the overall survival (OS) rates were reported to be $31 \%$ at 1 year, $7.9 \%$ at 2 years, and $0.9 \%$ at 4 years. ${ }^{8,9}$ Five-year survival was rare. However, the survival rate is still low if such patients are treated with chemotherapy only. ${ }^{9,10}$ Chemotherapy only achieves a median overall survival of 20 months despite optimal treatment and initial response. ${ }^{11}$

Local treatment strategies include surgical resection, radiofrequency ablation (RFA), and radiotherapy. ${ }^{12-14}$ The rationale for administering radiotherapy with a curative intent to patients with isolated RPLN metastases (IRM) should be the same as that in liver or lung metastasis.

Previous studies have shown that radiotherapy can be used for patients with IRM and could achieve good survival. Yeo et al analyzed 22 patients who received curative chemoradiotherapy. The 3- and 5-year OS rates were $64.7 \%$ and $36.4 \%$, respectively, and the median OS was 41 months. ${ }^{15}$

However, RPLN metastases are often accompanied by extra-retroperitoneal metastases (ERM) ${ }^{16}$ For patients with ERM, the role of radiotherapy is unclear. Radiotherapy for symptomatic colorectal cancer with ERM appears to provide relief of symptoms. ${ }^{17}$ While currently, there is no consensus on the role of radiotherapy for symptom relieving and disease control in patients with ERM.

The aim of this study is to evaluate the clinical effectiveness of radiotherapy for patients with IRM who may achieve NED and patients with symptomatic RPLN with ERM who aimed at relieving symptoms.

\section{Methods}

\section{Patients}

Colorectal cancer patients who received radiotherapy for RPLN metastases at West China hospital between March 2009 and November 2018 were retrospectively analyzed. Eligibility criterion: Eastern Cooperative Oncology Group performance status of 0 or 1 ; histologically confirmed adenocarcinoma of the colorectum; IRM which was defined as only retroperitoneal lymph node metastases were presented, without other metastases; or ERM which was defined as symptomatic RPLN metastases with stable or responded extra-retroperitoneal metastases in advanced colorectal cancer after systemic therapy; the radiation dose delivered to retroperitoneal lymphatic drainage area should be no less than 30Gy/15f; adequate bone marrow/liver/ kidney function.

The study was approved by the West China Hospital institutional review board. Our retrospective study was scrutinized without requiring consent from patients, because this study was low risk, anonymous, and conducted in accordance with the Declaration of Helsinki.

\section{Systematic Treatment}

After the diagnosis of RPLN metastases, all patients received chemotherapy with or without targeted therapy. Chemotherapy consisted of FOLFOX (5-fluorouracil plus oxaliplatin and leucovorin), FOLFIRI (5-fluorouracil plus irinotecan, and leucovorin), XELOX (capecitabine plus oxaliplatin). The chemotherapy regimens and cycles were decided by the treating oncologist or tumor board. Cetuximab or bevacizumab were given according to the genotype and the patients' wishes.

Radiotherapy could be determined to achieve NED or to relieve symptoms when stable or partial response of disease was obtained based on image revaluation after systematic treatment.

\section{Radiotherapy}

Patients were treated in the supine position with abdominal body thermoplastic masks. The helical computed tomography at $3 \mathrm{~mm}$ slice thickness with intravenous contrast was performed for every patient. The gross tumor volume (GTV) encompassed all involved lymph nodes observed on computed tomography (CT) or Positron Emission Tomography-Computed Tomography (PET-CT). The clinical target volume (CTV) was defined as the GTV and the locoregional nodal region including at least $2-3 \mathrm{~cm}$ extending from GTV in the superior-inferior direction. The planning target volume (PTV) was created by extending the CTV using a margin of $0.5 \mathrm{~cm}$. The PGTV incorporated a $0.5-\mathrm{cm}$ margin around the GTV, excluding areas adjacent to surrounding organ tissue. The radiation technique was administered using intensity modulated radiotherapy (IMRT) or Volumetric Arc Therapy Radiation Therapy (VMAT). A dose of 50-50.4 Gy/25-28 fractions was delivered to the PTV, followed by a boost of 10-16 Gy/5-8 fractions delivered to the PGTV. Plans were acceptable if the prescribed dose covered $>95 \%$ of the PTV and 
no more than $1 \mathrm{cc}$ received $>107 \%$ of the prescribed dose. Typical normal tissue constraints were as follows: spinal cord Dmax $<45$ Gy; $<40 \%$ of small bowel was to receive 30 Gy; small bowel Dmax $<54$ Gy; liver V30<33\%; one kidney Dmax $\leq 15$ Gy; each kidney Dmean $<15$ Gy. ${ }^{18}$

\section{Evaluation}

For the patients in the ERM group, the main symptom was pain before radiotherapy. The pain symptom was recorded on a $0-10$ numeric rating scale. Patients were evaluated to assess the degree of symptom relief during the course of radiotherapy and after the end of radiotherapy. The 3 levels of pain relief were defined as percentage relief $<30 \%$, percentage relief $30-70 \%$, and percentage relief $>70 \%$, respectively. ${ }^{18,19}$

After initial treatment, the patients underwent routine follow-up, including physical examination, laboratory tests, and abdominopelvic radiography every 3 months for the first 2 years and every 6 months thereafter. Colonovideoscopic examinations were conducted 1 year after treatment and then once every 2 years. When recurrent lesions were doubted, we implemented closer follow-up examinations including $\mathrm{CT}$ at a 1-month interval.

The response to treatment was evaluated based on CT scans after radiotherapy completed using the Response Evaluation Criteria in Solid Tumor (RECIST). ${ }^{20}$ A complete response (CR) was the reduction in short axis to $<10 \mathrm{~mm}$, a partial response (PR) was defined as a decrease of at least $30 \%$ in the sum of the short diameter of the treated lesions, progressive disease (PD) was an increase of at least $20 \%$ in the sum of the short diameter of the treated lesions or the appearance of new lesions, and stable disease (SD) was defined as neither a partial response nor progressive disease.

Treatment toxicity was assessed using the Common Terminology Criteria for Adverse Events version 3.0. The gastrointestinal (GI) toxicity was scored by clinical record form, as reported by the treating physician.

\section{Statistical Analysis}

The primary endpoints of this study were the overall survival (OS) and progression-free survival (PFS). The OS was defined as the time between the end of radiation and death from any cause. The PFS was defined as the time between the end of radiation and the first evidence of disease progression. Survival curves were calculated using the Kaplan-Meier method to access the outcome from the end of radiation. The secondary endpoints were treatment response, local control rate, and toxicities. The local control rate was defined as the proportion of patients free from the disease progression within the RT field during the follow-up time. A local recurrence was defined as a new lesion or disease progression within the RT field and distant recurrence was defined as a new lesion outside the retroperitoneum. Disease control (DCR) rate was defined as the proportion of patients with complete response, partial response, or stable disease.

The univariate analyses were used to test for differences in outcomes when stratifying by relevant factors. Multivariate analyses were performed for PFS and OS using the Cox proportional hazards model. A $p$-value of $<0.05$ was deemed statistically significant. All statistical tests were performed using SPSS software (release 20.0 IBM Corporation, USA).

\section{Results \\ Patient Characteristics}

Overall, 68 patients were enrolled in this study, of whom 28 (41\%) patients had ERM, and the other 40 patients had IRM. Patients' demographic and clinical characteristics are summarized in Table 1. Seven patients (17.5\%) in stage IV underwent a simultaneous resection of a solitary hepatic metastasis during the initial primary tumor surgery. The interval between original surgery for the primary colorectal cancer (CRC) and the diagnosis of retroperitoneal recurrence was 11 (range 1-62) months in the IRM group. The nature of the RPLNs is also summarized in Table 1.

More patients in the ERM group had RPLNs $>2 \mathrm{~cm}$ than in IRM group (64\% vs. $52.5 \%)$. In the IRM group, 6 patients (15\%) presented with pain, and 4 patients (10\%) presented with urinary obstruction. In the ERM group, 14 patients $(50 \%)$ presented with pain, 12 patients $(42.8 \%)$ presented with urinary obstruction, 2 patients $(7.2 \%)$ presented with liver metastases who can achieve NED for all metastases.

Most frequently, the extra-retroperitoneal metastases were located in the liver $(\mathrm{n}=17 ; 61 \%)$. The second most frequent extra-retroperitoneal metastases location was lungs $(n=7 ; 25 \%)$ and local recurrence $(n=4 ; 14 \%)$. Detailed information for each extra-retroperitoneal metastasis is provided in Table 2 .

\section{Treatment}

All patients received 5-fluorouracil based chemotherapy before radiotherapy. In the IRM group, chemotherapy consisted of FOLFIRI $(n=20)$, mFOLFOX6 $(n=9)$, and 
Table I Clinical and Pathologic Character of Patients

\begin{tabular}{|c|c|c|}
\hline Characteristics & IRM Group N=40 & ERM Group $N=28$ \\
\hline \multicolumn{3}{|l|}{ Age(years) } \\
\hline Median(range) & $58(30-78)$ & $54(28-66)$ \\
\hline \multicolumn{3}{|l|}{ Gender (\%) } \\
\hline Male & $21(52.5 \%)$ & $13(46 \%)$ \\
\hline Female & $19(47.5 \%)$ & $15(54 \%)$ \\
\hline \multicolumn{3}{|l|}{ ECOG performance status (\%) } \\
\hline 0 & $25(62.5 \%)$ & $2(7 \%)$ \\
\hline 1 & $15(37.5 \%)$ & $26(93 \%)$ \\
\hline \multicolumn{3}{|l|}{ Primary Tumor (\%) } \\
\hline Rectum & $8(20 \%)$ & $7(25 \%)$ \\
\hline Colon & $32(80 \%)$ & $21(75 \%)$ \\
\hline \multicolumn{3}{|l|}{ Initial TNM stage (\%) } \\
\hline ॥ & $8(20 \%)$ & $5(18 \%)$ \\
\hline III & $21(52.5 \%)$ & $13(46 \%)$ \\
\hline IV & $7(17.5 \%)$ & $9(32 \%)$ \\
\hline \multicolumn{3}{|l|}{ Tumor grade (\%) } \\
\hline G2: moderately differentiated & $18(45 \%)$ & $16(57 \%)$ \\
\hline G3: poorly differentiated & $12(30 \%)$ & $12(43 \%)$ \\
\hline \multicolumn{3}{|l|}{ RAS-BRAF mutations (\%) } \\
\hline \multicolumn{3}{|l|}{ BRAF } \\
\hline BRAF mutant & $2(5 \%)$ & 0 \\
\hline BRAF wild type & $16(40 \%)$ & $19(68 \%)$ \\
\hline Not done & $22(55 \%)$ & $9(32 \%)$ \\
\hline \multicolumn{3}{|l|}{ KRAS } \\
\hline KRAS mutant & $6(15 \%)$ & $7(25 \%)$ \\
\hline KRAS wild type & $\mathrm{II}(27.5 \%)$ & $11(39 \%)$ \\
\hline Not done & $21(52.5 \%)$ & $10(35.7 \%)$ \\
\hline \multicolumn{3}{|l|}{ NRAS } \\
\hline NRAS mutant & 0 & 0 \\
\hline NRAS wild type & $2(5 \%)$ & $4(14.3 \%)$ \\
\hline Not done & $38(95 \%)$ & $24(85.7 \%)$ \\
\hline \multicolumn{3}{|l|}{ Largest LN size (cm) } \\
\hline Range & $1.2-4.2$ & $1.3-5.8$ \\
\hline Median & 2.1 & 2.4 \\
\hline
\end{tabular}

Abbreviations: ERM, extra-retroperitoneal metastases; ECOG, Eastern Cooperative Oncology Group performance status; IRM, isolated retroperitoneal lymph node metastases; LN, lymph node; RAS-BRAF, RAS gene and BRAF gene.

XELOX $(n=11)$. In the ERM group, chemotherapy was given to 28 patients: FOLFIRI $(n=8), \operatorname{mFOLFOX6}(n=14)$, and XELOX $(n=6)$. Only 2 patients in the IRM group and 7 patients in the ERM group received targeted therapy before radiotherapy. All patients in the ERM group complete the radiotherapy. A median dose of 60 (range, 50-64.4) Gy was delivered in the IRM group. In the ERM group, only 2 patients failed to complete the radiotherapy due to the refusal and received the dose of $40 \mathrm{~Gy}$ and $44 \mathrm{~Gy}$, respectively. A median dose of 60 (range,
Table 2 Extra-Retroperitoneal Metastases Features

\begin{tabular}{|c|c|}
\hline Location & ERM Group \\
\hline Liver & $17(6 \mid \%)$ \\
\hline \multicolumn{2}{|l|}{ No. of metastases } \\
\hline Mean $\pm S D$ & $2 \pm 3$ \\
\hline Single liver mets & $13(46 \%)$ \\
\hline Radiotherapy to liver mets & $2(7 \%)$ \\
\hline Lung & $7(25 \%)$ \\
\hline \multicolumn{2}{|l|}{ No. of metastases } \\
\hline Mean \pm SD & $I \pm 6$ \\
\hline Single lung mets & $4(14 \%)$ \\
\hline Radiotherapy to lung mets & 0 \\
\hline Local recurrence & $4(14 \%)$ \\
\hline Radiotherapy to local recurrence & $3(10 \%)$ \\
\hline Other sites & $6(21 \%)$ \\
\hline Two sites of metastases & $6(21 \%)$ \\
\hline Pain & $14(50 \%)$ \\
\hline Ureteral obstruction & $12(43 \%)$ \\
\hline
\end{tabular}

Abbreviations: ERM, extra-retroperitoneal metastases; Mets, metastases; SD, standard deviation.

40-66) Gy was delivered in the ERM group. Concurrent chemotherapy consisting of capecitabin $(n=8)$ and XELOX $(n=8)$ was delivered in 7 patients in the IRM group and 9 patients in the ERM group. In the ERM group, 2 patients who can achieve NED underwent concomitant local therapy for extra-retroperitoneal metastases including radiotherapy or RFA for liver metastases, of which 1 patient eventually achieved NED. To relieve the urinary obstruction, an internal ureteral stent was placed in 2 patients in both the IRM group and the ERM group. In the ERM group, 1 patient was treated by percutaneous nephrostomy.

Only 18 patients in the IRM group and 19 patients in the ERM group underwent genetic testing. KRAS mutation and BRAF mutation were detected in 6 and 2 patients in the IRM group, and 7 and 0 in the ERM group, respectively. MMR defects were detected in 2 patients in both groups. Bevacizumab was used in 8 patients $(20 \%)$ in the IRM group and 5 patients (18\%) in the ERM group, respectively. Five patients were treated with cetuximab in both groups.

\section{Response}

In the IRM group, a complete treatment response was found in $5(12.5 \%)$ patients, $20(50 \%)$ patients showed a partial response, and $9(22.5 \%)$ patients had stable disease. The 1-, 2-, and 3-year local control rates were $87.5 \%$, 
$77.5 \%$, and $70 \%$, respectively. The number of patients with $<30 \%, 30-70 \%$, and $\geq 70 \%$ pain relief was 2 $(33.3 \%), 2(33.3 \%)$, and $2(33.3 \%)$, respectively. Four patients $(100 \%)$ had a complete relief in urinary obstruction.

In the ERM group, 1 patient (3.6\%) showed complete response in both RPLN and extra-retroperitoneal metastases, a partial response was found in 4 patients (14.2\%), and 16 patients $(57.1 \%)$ had stable disease. The local control rates for the ERM group at 1, 2, and 3 years were $57.1 \%, 42.8 \%$, and $0 \%$, respectively.

The number of patients with $<30,30-70 \%$, and $\geq 70 \%$ pain relief was $2(14.3 \%), 4(28.6 \%)$, and $6(42.9 \%)$, respectively. Two patients did not undergo pain evaluation. Overall, $11(91.7 \%)$ patients had a complete $(n=5)$ or partial relief $(n=6)$ in urinary obstruction, 1 patient had progressive urinary obstruction.

\section{Survival}

The median follow-up for the entire study population was 38 (range, 5-119) months. Median OS and PFS were 59.4 months and 22.9 months in the IRM group, and 19.7 months and 3.7 months in the ERM group, respectively.
The OS and PFS were significantly lower for patients in the ERM group compared with the IRM group $(p<0.05$, Figure $1 ; p<0.05$, Figure 2), respectively.

\section{Prognostic Factors}

The univariate analyses indicated that in the IRM group, CR or PR were associated with better OS. The $\mathrm{N}$ category $<2, \mathrm{CR}$ or $\mathrm{PR}, \mathrm{LN}$ size $\leq 2 \mathrm{~cm}$, and interval $\leq 12$ months were favorable prognostic factors for PFS (Table 3). The multivariate analysis also showed that treatment response was a significant factor for OS and the $\mathrm{N}$ category $<2$, treatment response and interval $\leq 12$ months were significant factors for PFS. Patients with CR and PR had a 3-year OS of $92.9 \%$, while patients with SD and PD had a 3 -year OS of $30 \%(p<0.05)$. The 3 -year PFS for patients with N0-1 was $47.9 \%$ and for patients with $\mathrm{N} 2$ was $0 \%(p<0.05)$. Other factors including age, sex, primary tumor site, genotype, and targeted therapy did not demonstrate statistically significant effects (Table 3).

In the ERM group, $\mathrm{N}$ category $<2$, infrarenal lymph node and isolated liver metastases were statistically significant factors affecting PFS according to univariate

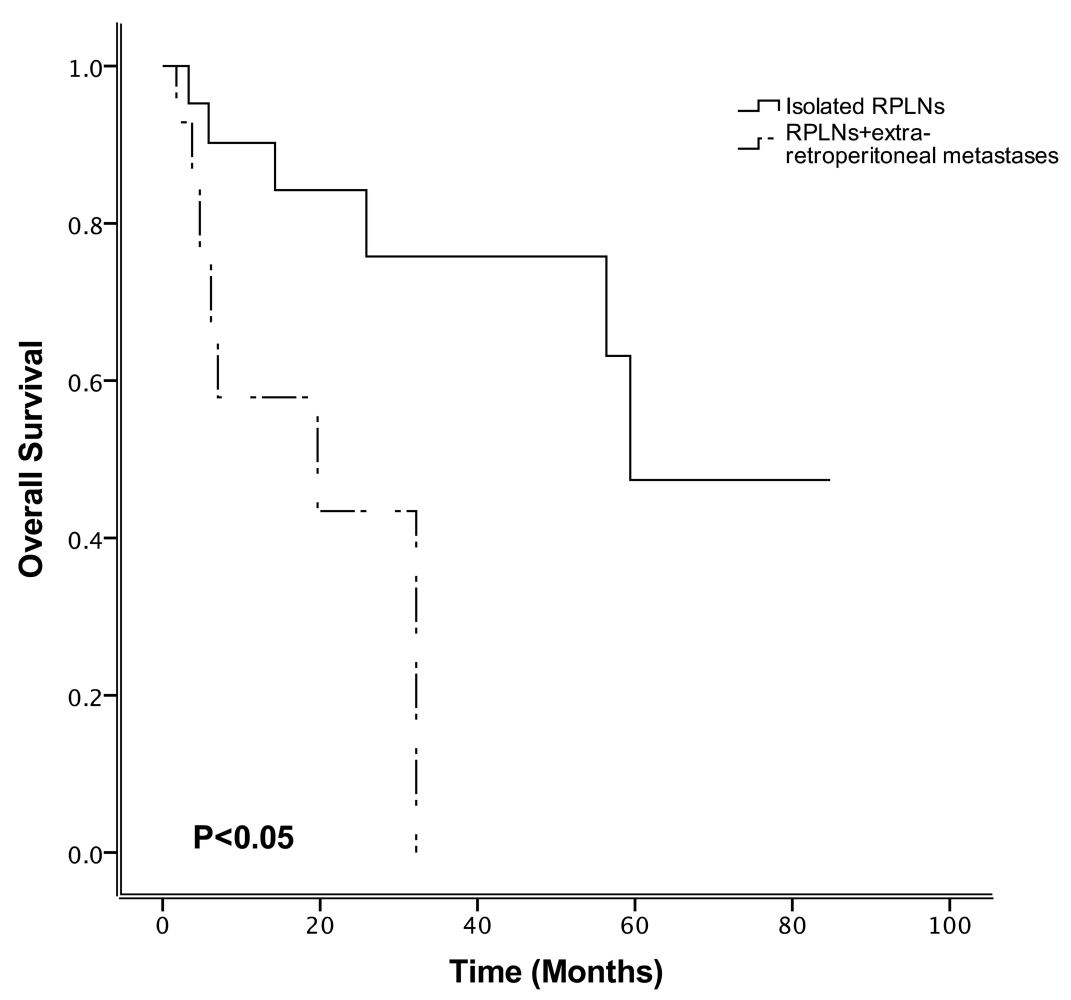

Figure I Overall survival according to the presence or absence of extra-retroperitoneal metastases within the total study population. Abbreviation: RPLN, retroperitoneal lymph node. 


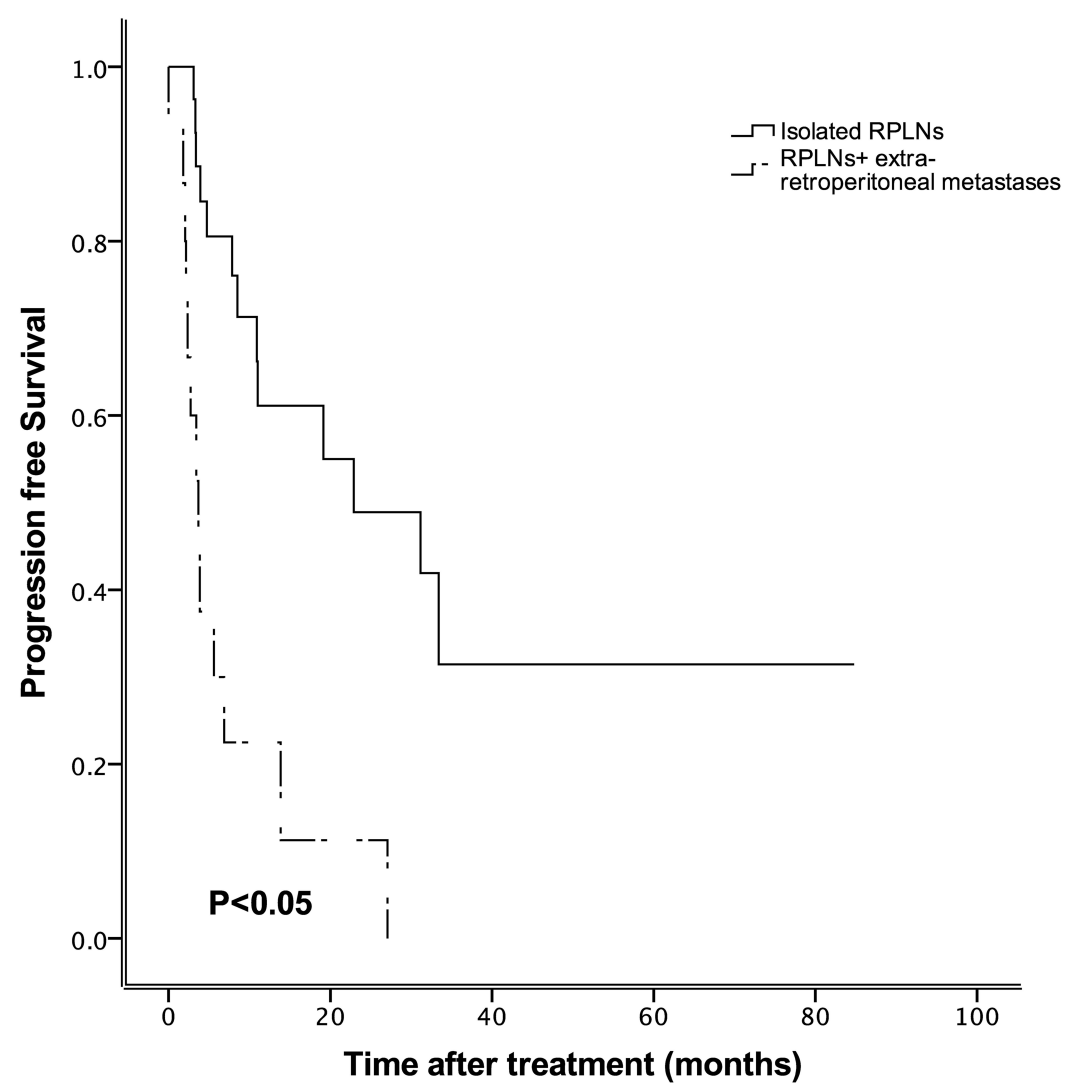

Figure 2 Progression-free survival according to the presence or absence of extra-retroperitoneal metastases within the total study population. Abbreviation: RPLN, retroperitoneal lymph node.

analyses (Table 4). The multivariate analysis indicated that the infrarenal lymph node site and isolated liver metastases remained a statistically significant factor affecting PFS. The 1-year PFS for patients with infrarenal lymph node was $38.1 \%$ and for patients with suprarenal lymph node was $0 \%(p<0.05)$.

\section{Toxicities}

Radiotherapy was well tolerated with only 3 patients experiencing grade 3 hematologic toxicities (4.4\%) including leukopenia or thrombocytopenia and 2 patients undergoing grade 3 liver injury (3\%). During radiotherapy, 5 patients $(7.4 \%)$ developed acute gastrointestinal (GI) toxicities such as nausea and diarrhea, including 1 patient with grade-3 toxicity.

\section{Discussion}

Retroperitoneal lymph nodal metastases are rare and have poor survival outcomes. There has been no consensus on therapeutic strategy. The aim of our study was to explore the safety and efficacy of radiotherapy for RPLNs in colorectal cancer patients.
It was recommended by the EMSO that in patients with oligometastatic disease, the goal of NED should be achieved. ${ }^{7}$ Several studies have suggested that surgery as a method to achieve NED for isolated RPLNs can achieve long-term OS (34 40 months) in selected patients with acceptable postoperative morbidity. ${ }^{6,8}$ Radiotherapy can be used as an alternative to surgery to achieve NED. Our results suggest that radiotherapy is acceptably safe and efficient.

Meanwhile, radiotherapy can be used as palliative treatment in patients with symptoms and extensive metastases. According to our study, palliative radiotherapy for symptomatic RPLNs appears to provide relief of symptoms, although the survival in this group is poor.

A few retrospective trials have been published in recent years evaluating the benefit of radiotherapy in patients with isolated RPLNs. ${ }^{11,21} \mathrm{Kim}$ et al detailed the outcomes for 7 patients with isolated RPLN metastases who were treated between 2003 and 2009 using stereotactic body radiotherapy with a dose ranged from 36 Gy to 51 Gy. The 1 year and 3 year overall survival rates were $100 \%$ and $71.4 \%$, respectively, and median survival was 37 months. A Korean retrospective study applied concurrent chemoradiotherapy to isolated 
Table 3 Univariate and Multivariate Cox Regression Analyses for PFS and OS in Isolated RPLNs

\begin{tabular}{|c|c|c|c|c|}
\hline & UVA PFS & MVA PFS & UVA OS & MVA OS \\
\hline & HR 95\% CI P & HR 95\% CI P & HR 95\% CI P & HR 95\% CI P \\
\hline Age, year $(\geq 50$ vs. $<50)$ & $1.630 .44-5.990 .47$ & $\mathrm{NI}$ & $0.7|0.1|-4.420 .7 \mid$ & $\mathrm{NI}$ \\
\hline Sex (male vs female) & $1.570 .52-4.720 .42$ & $\mathrm{NI}$ & $3.210 .57-17.90 .19$ & $\mathrm{NI}$ \\
\hline Primary site (colon vs rectum) & $0.570 .15-2.110 .39$ & $\mathrm{NI}$ & $0.370 .04-3.250 .37$ & $\mathrm{NI}$ \\
\hline Tumor grade (G3 vs G2) & $0.290 .07-1.230 .09$ & $0.380 .08-1.790 .16$ & $0.240 .04-1.460 .12$ & $0.100 .01-1.110 .06$ \\
\hline T-category ( $\leq \mathrm{T} 3$ vs $>\mathrm{T} 4)$ & $1.790 .61-5.330 .29$ & $\mathrm{NI}$ & $1.090 .25-4.770 .91$ & $\mathrm{NI}$ \\
\hline N-category (N2 vs N0-I) & $0.190 .05-0.710 .01$ & $0.180 .04-0.790 .02$ & $0.110 .01-1.070 .05$ & $0.140 .01-1.310 .08$ \\
\hline RAS and BRAF status (wild type vs mutant type) & $0.610 .10-3.740 .59$ & $\mathrm{NI}$ & $1.270 .07-21.80 .87$ & $\mathrm{NI}$ \\
\hline Interval, months (>12 vs $\leq 12)$ & $0.240 .06-0.890 .03$ & $0.140 .02-0.880 .04$ & $0.270 .05-1.570 .15$ & $0.310 .07-1.680 .31$ \\
\hline LN site (suprarenal vs infrarenal) & $1.090 .36-3.270 .88$ & $\mathrm{NI}$ & $3.980 .46-34.40 .21$ & $\mathrm{NI}$ \\
\hline LN size $(\leq 2 \mathrm{~cm}$ vs $>2 \mathrm{~cm})$ & $4.87 \quad 1.15-20.60 .03$ & $9.040 .97-83.50 .05$ & $0.52 \quad 0.05-5.080 .57$ & $\mathrm{NI}$ \\
\hline Radiation dose ( $<50$ Gy vs $\geq 50$ Gy) & $0.310 .07-1.280 .10$ & $0.080 .01-1.260 .11$ & $0.330 .06-1.880 .21$ & $\mathrm{NI}$ \\
\hline RECIST (CR+PR vs SD+PD) & $5.05 \quad 1.55-16.50 .01$ & $5.81 \quad 1.17-29.00 .03$ & $13.1 \quad 1.46-1180.02$ & $22.0 \quad 1.58-3080.02$ \\
\hline Targeted therapy (Yes vs No) & $0.860 .26-2.860 .81$ & $\mathrm{NI}$ & $2.450 .29-2 \mathrm{I} . \mathrm{I} 0.4 \mathrm{I}$ & $\mathrm{NI}$ \\
\hline Targeted drug (cet vs bev) & $0.740 .07-8.4 \mid 0.8$ I & $\mathrm{NI}$ & $0.010-5000.58$ & $\mathrm{NI}$ \\
\hline
\end{tabular}

Abbreviations: bev, bevacizumab; $\mathrm{Cl}$, confidence interval; CR, complete response; cet, cetuximab; HR, hazard ratio; LN, lymph node; MVA, multivariate analyses; mutant type, RAS-BRAF mutant; N, lymph node; NI, not included; OS, overall survival; PFS, progression-free survival; PR, partial response; PD, progressive disease; RPLN, retroperitoneal lymph node; RECIST, Response Evaluation Criteria in Solid Tumor; SD, stable disease; T, tumor; UVA, univariate analyses; wild type, RAS wild type.

Table 4 Univariate and Multivariate Cox Regression Analyses for PFS and OS in ERM Group

\begin{tabular}{|c|c|c|c|c|}
\hline & UVA PFS & MVA PFS & UVA OS & MVA OS \\
\hline & HR 95\% Cl P & HR 95\% CI P & HR 95\% CI P & HR 95\% CI P \\
\hline Age, year $(\geq 50$ vs $<50)$ & $\mathrm{I} .740 .5 \mathrm{I}-5.980 .38$ & $\mathrm{NI}$ & $0.490 .05-4.400 .53$ & $\mathrm{NI}$ \\
\hline Sex (male vs female) & $0.520 .16-1.660 .27$ & $\mathrm{NI}$ & $0.990 .18-5.470 .99$ & $\mathrm{NI}$ \\
\hline Primary site (colon vs rectum) & $0.830 .26-2.630 .75$ & $\mathrm{NI}$ & $1.110 .20-6.120 .91$ & $\mathrm{NI}$ \\
\hline Tumor grade (G3 vsG2) & $1.910 .60-6.090 .27$ & $\mathrm{NI}$ & $0.840 .15-4.640 .84$ & $\mathrm{NI}$ \\
\hline T-category ( $\leq \mathrm{T} 3$ vs $>\mathrm{T} 4)$ & $0.360 .10-1.290 .12$ & $0.400 .12-1.340 .10$ & $0.580 .09-3.7 \mid 0.58$ & $\mathrm{NI}$ \\
\hline $\mathrm{N}$-category (N2 vs N0-I) & $0.220 .05-0.920 .04$ & $0.220 .05-0.920 .05$ & $0.340 .06-2.090 .24$ & $\mathrm{NI}$ \\
\hline RAS and BRAF status (wild type vs mutant type) & $1.260 .29-5.330 .75$ & $\mathrm{NI}$ & $5.150 .53-49.80 .16$ & $\mathrm{NI}$ \\
\hline Interval, months (>12 VS. $\leq 12)$ & $0.780 .23-2.670 .69$ & $\mathrm{NI}$ & $1.630 .27-9.850 .59$ & $\mathrm{NI}$ \\
\hline LN site(suprarenal vs. infrarenal) & $0.090 .02-0.490 .01$ & $0.10 .02-0.550 .01$ & $0.390 .07-2.240 .29$ & $\mathrm{NI}$ \\
\hline LN size $(\leq 2$ vs $>2)$ & $0.760 .23-2.490 .65$ & $\mathrm{NI}$ & $2.170 .37-12.50 .39$ & $\mathrm{NI}$ \\
\hline Radiation dose(<50 Gy vs $\geq 50$ Gy) & $0.310 .07-1.260 .10$ & $0.340 .08-1.320 .11$ & $0.030 .00-44.60 .34$ & $\mathrm{NI}$ \\
\hline Targeted therapy (Yes vs No) & $1.080 .32-3.670 .90$ & $\mathrm{NI}$ & $0.820 .09-7.180 .86$ & $\mathrm{NI}$ \\
\hline Targeted drug (cet vs bev) & $0.640 .15-2.740 .55$ & $\mathrm{NI}$ & I.17 0.18-7.490.87 & $\mathrm{NI}$ \\
\hline Extra-retroperitoneal metastases & & & & \\
\hline Isolated lung metastases (yes vs no) & $2.160 .46-10.10 .33$ & $\mathrm{NI}$ & $1.890 .21-17.10 .57$ & $\mathrm{NI}$ \\
\hline Isolated liver metastases (yes vs no) & $0.200 .06-0.740 .02$ & $0.230 .06-0.860 .03$ & $0.270 .05-1.5 \mid 0.14$ & $\mathrm{NI}$ \\
\hline Number of ERM sites $(\leq 1$ vs $>1)$ & I.46 $0.39-5.570 .58$ & $\mathrm{NI}$ & $0.530 .06-4.490 .56$ & $\mathrm{NI}$ \\
\hline
\end{tabular}

Abbreviations: bev, bevacizumab; $\mathrm{Cl}$, credibility interval; $\mathrm{CR}$, complete response; cet, cetuximab; ERM, extra-retroperitoneal metastases; HR, hazard ratio; LN, lymph node; MVA, multivariate analyses; mutant type, RAS-BRAF mutant; N, lymph node; NI, not included; OS, overall survival; PFS, progression-free survival; PR, partial response; PD, progressive disease; RECIST, Response Evaluation Criteria in Solid Tumor; SD, stable disease; T, tumor; UVA, univariate analyses; wild type, RAS wild type.

retroperitoneal lymph node recurrence of colorectal cancer. It is shown that chemoradiotherapy is an effective and safe salvage treatment for isolated retroperitoneal lymph node recurrence. The radiotherapy technique used in their study was three-dimensional conformal RT or helical with a total dose of 63 Gy in 35 fractions or 55.8 Gy in 31 fractions. ${ }^{15}$
Furthermore, a retrospective Japanese study employed the carbon-ion radiotherapy for isolated para-aortic lymph node recurrence. It was reported that the radiotherapy improved the local control and survival rates in the absence of chemotherapy. The overall survival rates at 2 and 3 years were $83.3 \%$ and $63.0 \%$, respectively. ${ }^{22}$ In the Korean study, where patients 
were treated with three-dimensional conformal radiotherapy or helical tomotherapy plus capecitabine or 5-fluorouracil, it was reported that the 3- and 5-year overall survival rates were $64.7 \%$ and $36.4 \%$, median overall survival was 41 months and median recurrence-free survival was 20 months. In our study, we used the IMRT or VMAT to further explore the safety and efficacy of radiotherapy combined with systemic therapy. When compared with the results of those four reports above, our results are comparable. Within our series, the median OS was 59.4 months. The 1-year and 3-year OS were 90.2\%, $75.8 \%$. The 1 -year and 3-year PFS were $57.9 \%$ and $0 \%$, respectively.

Lee et al reported that low-dose radiation and a tumor location above the renal vein were both independent risk factors for local recurrence. ${ }^{23}$ In the study reported by Yeo et al, response and adjuvant chemotherapy were significant prognostic factors for overall survival. ${ }^{15}$ In our study, the results of the multivariate analysis revealed that treatment response was a significant factor affecting $\mathrm{OS}$, and $\mathrm{N}$ category $<2, \mathrm{LN}$ size $\leq 2 \mathrm{~cm}$, and interval $\leq 12$ months were significant factors for PFS was a statistically significant factor affecting only PFS.

Little is known about the effect of palliative radiotherapy to RPLNs in CRC patients with other systemic metastases. However, it was reported that local treatment to the retroperitoneal area could be useful when the patients presented with RPLNs and one extra-retroperitoneal metastasis such as pulmonary metastases or liver metastases. ${ }^{10,24-26}$ In our study, those patients with symptomatic RPLNs and extraretroperitoneal metastases undergoing radiotherapy to retroperitoneal lymph nodal metastases were enrolled. Radiotherapy is a very good choice for pain palliation and urinary tract obstruction palliation, although it did not improve the survival rate. The local control rate was found to be higher in the IRM group compared with the ERM group. This is probably due to the larger lymph nodal size and more aggressive biological behavior in the ERM group. $^{27,28}$ The patients with infrarenal lymph node have a better prognosis in the ERM group.

Our study has several limitations due to its retrospective nature. A deviation of analysis results may result from the small sample sizes, different baselines of patients, and different treatment regimens. Univariate analyses on small samples incur a substantial risk of type II error.

In conclusion, our analysis suggests radiotherapy aimed at achieving NED is a safe and effective treatment for IRM. OS and PFS were longer in patients with CR and PR compared with patients with SD and PD. Radiotherapy can also be used as palliative treatment for symptomatic RPLNs in ERM. Larger-scale research assessing the effects of radiotherapy in recurrent colorectal cancer is needed.

\section{Disclosure}

The authors declare no conflicts of interest and no funding received for this study.

\section{References}

1. Favoriti P, Carbone G, Greco M, et al. Worldwide burden of colorectal cancer: a review. Updates Surg. 2016;68(1):7-11. doi:10.1007/ s13304-016-0359-y

2. Brenner H, Kloor M, Pox CP. Colorectal cancer. Lancet. 2014;383 (9927):1490-1502. doi:10.1016/S0140-6736(13)61649-9

3. Lee WS, Yun SH, Chun HK, et al. Pulmonary resection for metastases from colorectal cancer: prognostic factors and survival. Int J Colorectal Dis. 2007;22(6):699-704. doi:10.1007/s00384-0060218-2

4. Fong Y, Cohen AM, Fortner JG, et al. Liver resection for colorectal metastases. J Clin Oncol. 1997;15(3):938-946. doi:10.1200/ JCO.1997.15.3.938

5. Choi PW, Kim HC, Kim AY, et al. Extensive lymphadenectomy in colorectal cancer with isolated para-aortic lymph node metastasis below the level of renal vessels. J Surg Oncol. 2010;101(1):66-71. doi:10.1002/jso.21421

6. Min BS, Kim NK, Sohn SK, et al. Isolated paraaortic lymph-node recurrence after the curative resection of colorectal carcinoma. J Surg Oncol. 2008;97(2):136-140. doi:10.1002/jso.20926

7. Van Cutsem E, Cervantes A, Adam R, et al. ESMO consensus guidelines for the management of patients with metastatic colorectal cancer. Ann Oncol. 2016;27(8):1386-1422. doi:10.1093/annonc/mdw235

8. Biasco G, Derenzini E, Grazi G, et al. Treatment of hepatic metastases from colorectal cancer: many doubts, some certainties. Cancer Treat Rev. 2006;32(3):214-228. doi:10.1016/j.ctrv.2005.12.011

9. Saltz LB. Metastatic colorectal cancer: is there one standard approach? Oncology. 2005;19(9):1147-54; discussion 54, 57-8, 60.

10. Ho TW, Mack LA, Temple WJ. Operative salvage for retroperitoneal nodal recurrence in colorectal cancer: a systematic review. Ann Surg Oncol. 2011;18(3):697-703. doi:10.1245/s10434-010-1322-7

11. Kim M-S, Cho CK, Yang KM, et al. Stereotactic body radiotherapy for isolated paraaortic lymph node recurrence from colorectal cancer. World J Gastroenterol. 2009;15(48):6091-6095. doi:10.3748/wjg.15.6091

12. Van Cutsem E, Nordlinger B, Adam R, et al. Towards a pan-European consensus on the treatment of patients with colorectal liver metastases. Eur J Cancer. 2006;42(14):2212-2221. doi:10.1016/ j.ejca.2006.04.012

13. Dhir M, Sasson AR. Surgical management of liver metastases from colorectal cancer. J Oncol Pract. 2016;12(1):33-39. doi:10.1200/ JOP.2015.009407

14. Gonzalez M, Poncet A, Combescure C, et al. Risk factors for survival after lung metastasectomy in colorectal cancer patients: a systematic review and meta-analysis. Ann Surg Oncol. 2013;20(2):572-579. doi:10.1245/s10434-012-2726-3

15. Yeo S-G, Kim DY, Kim TH, et al. Curative chemoradiotherapy for isolated retroperitoneal lymph node recurrence of colorectal cancer. Radiother Oncol. 2010;97(2):307-311. doi:10.1016/j.radonc.2010. 05.021

16. Hashimoto M, Komatsu H, Naruse Y, et al. Resection of paraaortic lymph node metastasis of colon cancer with graft replacement. Hepato Gastroenterol. 2003;50(51):709-710. 
17. Cameron MG, Kersten C, Vistad I, et al. Palliative pelvic radiotherapy of symptomatic incurable rectal cancer - a systematic review. Acta Oncol. 2014;53(2):164-173. doi:10.3109/0284186X.2013. 837582

18. Marks LB, Ten Haken RK, Martel MK. Guest editor's introduction to QUANTEC: a users guide. Int J Radiat Oncol Biol Phys. 2010;76(3 Suppl):S1-S2. doi:10.1016/j.ijrobp.2009.08.075

19. Agarwal JP, Nemade B, Murthy V, et al. Hypofractionated, palliative radiotherapy for advanced head and neck cancer. Radiother Oncol. 2008;89(1):51-56. doi:10.1016/j.radonc.2008.06.007

20. Therasse P, Arbuck SG, Eisenhauer EA, et al. New guidelines to evaluate the response to treatment in solid tumors. European Organization for Research and Treatment of Cancer, National Cancer Institute of the United States, National Cancer Institute of Canada. J Natl Cancer Inst. 2000;92(3):205-216.

21. Johnson B, Jin Z, Haddock MG, et al. A curative-intent trimodality approach for isolated abdominal nodal metastases in metastatic colorectal cancer: update of a single-institutional experience. Oncologist. 2018;23(6):679-685. doi:10.1634/theoncologist.2017-0456

22. Isozaki Y, Yamada S, Kawashiro S, et al. Carbon-ion radiotherapy for isolated para-aortic lymph node recurrence from colorectal cancer. J Surg Oncol. 2017;116(7):932-938. doi:10.1002/jso.24757

23. Lee J, Chang JS, Shin SJ, et al. Incorporation of radiotherapy in the multidisciplinary treatment of isolated retroperitoneal lymph node recurrence from colorectal cancer. Ann Surg Oncol. 2015;22 (5):1520-1526. doi:10.1245/s10434-014-4363-5
24. Gagniere J, Dupre A, Chabaud S, et al. Retroperitoneal nodal metastases from colorectal cancer: curable metastases with radical retroperitoneal lymphadenectomy in selected patients. Eur J Surg Oncol. 2015;41(6):731-737. doi:10.1016/j.ejso.2015.03.229

25. Taylor WE, Donohue JH, Gunderson LL, et al. The mayo clinic experience with multimodality treatment of locally advanced or recurrent colon cancer. Ann Surg Oncol. 2002;9(2):177-185. doi:10.1007/BF02557371

26. Wong JS, Tan GH, Teo MC. Management of para-aortic lymph node metastasis in colorectal patients: a systemic review. Surg Oncol. 2016;25(4):411-418. doi:10.1016/j.suronc.2016.09.008

27. Evans MD, Escofet X, Karandikar SS, Stamatakis JD. Outcomes of resection and non-resection strategies in management of patients with advanced colorectal cancer. World J Surg Oncol. 2009;7(1):28. doi:10.1186/1477-7819-7-28

28. Eisenberger A, Whelan RL, Neugut AI. Survival and symptomatic benefit from palliative primary tumor resection in patients with metastatic colorectal cancer: a review. Int J Colorectal Dis. 2008;23 (6):559-568. doi:10.1007/s00384-008-0456-6

\section{Publish your work in this journal}

Cancer Management and Research is an international, peer-reviewed open access journal focusing on cancer research and the optimal use of preventative and integrated treatment interventions to achieve improved outcomes, enhanced survival and quality of life for the cancer patient.
The manuscript management system is completely online and includes a very quick and fair peer-review system, which is all easy to use. Visit http://www.dovepress.com/testimonials.php to read real quotes from published authors. 